\title{
Fitting Direct Interaction Pieces into Neutrino Puzzles
}

\author{
Loretta M. Johnson ${ }^{1}$ and Douglas W. McKay ${ }^{2}$ \\ Department of Physics and Astronomy, University of Kansas, Lawrence, KS \\ 66045, USA
}

\begin{abstract}
We develop a framework and unified, compact notation to include neutrino direct interaction effects with neutrino oscillations for a wide class of lowenergy, effective four-Fermi interactions. Modest flavor-violating interaction strengths can make significant changes in the boundaries in $\Delta M^{2} \leftrightarrow \sin ^{2} 2 \theta$ and in $\tan ^{2} \theta_{13} \leftrightarrow \tan ^{2} \theta_{23}$ parameter space. We draw examples from the $L-R$ symmetric and $M S S M+\not R$ models and find the recently reported decay-at-rest $\mu \leftrightarrow e$ transition probability can be described without oscillations in $L-R$ symmetric models.
\end{abstract}

PACS: 13.15.+g, 14.60.Pq, 12.60.-i, 12.60.Jv. Keywords: neutrino, oscillation; neutrino, flavor; muon+, leptonic decay; pi+, leptonic decay; interaction, neutrino $\mathrm{p}$; numerical calculations, interpretation of experiments.

\section{Introduction}

Reconciling the solar [1], atmospheric [2], and accelerator[3, 4, 5] neutrino data is difficult within a purely 3-family oscillation description, which provides for two mass-squared differences, 3 mixing angles and a $\mathrm{CP}$ violating phase [6, 8, 8]. With this in mind we develop a unified framework to combine the oscillation new physics with, presumably related, direct-interaction new physics. We apply the general formalism to a number of cases with an eye to accommodating all of the positive signals for new phenomena within a three-family framework. We focus mainly on the picture where the favored[9] $\nu_{e} \leftrightarrow \nu_{\mu} \mathrm{MSW}$ [10] oscillation is the solution to the solar neutrino observations, a $\nu_{\mu} \leftrightarrow \nu_{\tau}$ oscillation is the solution to the atmospheric neutrino anomaly 11 and a direct interaction of the type $\nu_{\mu}+n \rightarrow e^{-}+p$ at the detector and/or $\pi^{+} \rightarrow \mu^{+}+\nu_{e}$ or $\mu^{+} \rightarrow e^{+}+\bar{\nu}_{e}+\nu_{\mu}$ at the source contribute substantially to the LSND signal [3, 迎 for the " $\nu_{e}$ appearance" at the few tenths of a percent level. The direct interaction effects are flightpath independent and, moreover, they are clearly too small to explain the large solar and atmospheric anomalies that have been reported. At the same time, the strictly two-flavor $\nu_{e} \leftrightarrow \nu_{\mu}$ oscillations with currently preferred[9] mixing and mass values, are negligible in the short-baseline experiments of the LSND

\footnotetext{
${ }^{1}$ Email address: johnson@kuphsx.phsx.ukans.edu

${ }^{2}$ Email address: mckay@kuphsx.phsx.ukans.edu
} 
type. To complement the "two two-flavor", $\nu_{e} \leftrightarrow \nu_{\mu}$ plus $\nu_{\mu} \leftrightarrow \nu_{\tau}$, picture just mentioned, we include a brief discussion of a simple "one-scale" version of three-flavor mixing within our framework [6]. We show the addition of modest direct interaction effects extends the allowed parameter space in an interesting way.

A clean and convenient parameterization of the situation can be achieved by separating the problem into the production (source), propagation (with or without matter enhancement effects) and observation (detector) components 12 , 13, 14. First we introduce a working phenomenological model which includes lepton-flavor violating, four-Fermi interactions of purely leptonic and of semileptonic types. [3 Next we present the setup to describe the production, propagation and detection and define the "appearance probability" for the situation where the neutrinos are relativistic, the source is a muon or charged pion, the propagation is in vacuum and the detector is a neutron or proton (or nucleus) target. We then survey serveral patterns of dominant interactions suggested by the data. We compare these patterns with those presented by the minimal left-right symmetric model 15, 16] and the minimal supersymmetric model augmented by a minimal set of discrete R-parity violating terms 17, 18. Considering the LSND 3, [1 result by itself, we make a study of the shift in allowed mass and mixing angle parameter space that is afforded by including direct interactions. In our concluding discussion, we point out several open possibilities.

\section{A Working Model}

To treat the bulk of the current accelerator data and to illustrate the general ideas, it suffices to take a model with two lepton families and one quark family with charged current, effective four-Fermi interactions. The model semileptonic (S) and leptonic (L) effective Lagrangian takes the form $L_{e f f}=L^{S}+L^{L}$, wheref

$$
\begin{gathered}
L^{S}=2^{3 / 2} G_{F} O_{A}\left(K_{A i j}^{h} \bar{l}_{i} \Gamma_{A} P_{h} U_{j a} \nu_{a}\right)+h . c . \\
L^{L}=2^{3 / 2} G_{F} F_{A i j k m}^{h} \bar{l}_{i} \Gamma_{A} P_{h} U_{j a} \nu_{a}\left(\bar{l}_{k} \Gamma_{A} P_{h} U_{m b} \nu_{b}\right)^{\dagger},
\end{gathered}
$$

where the coefficients $\mathrm{K}$ and $\mathrm{F}$ give the amount of admixture of $h=L, R$ projection, the type of Dirac matrix structure and the lepton flavors contained in $L_{\text {eff }}$. Possible crossed terms between $L$ and $R$ give contributions to the unpolarized cross sections that are proportional to $m_{\nu}$ 's and we ignore these effects here. The hadronic operators are of form $O_{A}=\bar{d} \Gamma_{A}\left(a P_{L}+b P_{R}\right) u$. All repeated indices are summed: $A=S, V, T$ (matching Dirac gamma matrices

\footnotetext{
${ }^{3}$ The questions of the detailed form and origin of the neutrino mass matrix are not addressed here.

${ }^{4}$ The factor of $\frac{1}{2}$ in the definitions $P_{L}=\frac{1}{2}\left(1-\gamma_{5}\right)$ and $P_{R}=\frac{1}{2}\left(1+\gamma_{5}\right)$ leads to the factor $2^{3 / 2}$ when combined with the conventional $\frac{G_{F}}{\sqrt{2}}$ factor in the four-Fermi interactions. Neutral current SM terms are also contained the $L^{L}$ term by Fierz symmetry of $(V-A) \times(V-A)$.
} 
Table 1: Some relevant expressions for $K$ and $F$ coefficients in the SM, $L-R$ and $M S S M+\not R$ models. The $L-R$ and $\not R$ entries should be divided by $G_{F} 2^{3 / 2}$.

\begin{tabular}{|c|c|c|c|c|c|}
\hline & $K_{V i i}^{L}$ & $K_{V 12}^{L}$ & $K_{S 12}^{L}$ & $F_{V 1122}^{L}$ & $F_{V 211 m}$ \\
\hline$S M$ & 1 & 0 & 0 & 1 & 0 \\
\hline$L-R$ & 0 & 0 & 0 & $\frac{h_{21} h_{12}^{*}}{M_{\Delta_{L}}^{2}}$ & $\frac{h_{11} h_{2 m}^{*}}{M_{\Delta_{L}}^{2}}$ \\
\hline$\not R$ & $\sum_{k} \frac{\left|\lambda_{i j k}^{\prime}\right|^{2}}{M_{\tilde{d}_{R}^{k}}^{2}}$ & $\sum_{k} \frac{\lambda_{21 j}^{\prime} \lambda_{11 k}^{\prime}}{M_{\tilde{d}_{R}^{k}}^{2}}$ & $\frac{\lambda_{311}^{\prime} \lambda_{231}}{M_{\tilde{e}_{3}}^{2}}$ & $\frac{-\left|\lambda_{123}\right|^{2}}{2 M_{\tilde{e}_{R}^{3}}^{2}}$ & 0 \\
\hline
\end{tabular}

$\mathbf{1}, \gamma$ and $\sigma), h=L, R$, lepton flavor indices $i, j, k, m$ and mass eigenstate indices $a, b$. The $U_{j a}$ are the unitary transformation matrices between the flavor states and the mass eigenstates.

In a two-flavor mixing scheme where $\nu_{e}$ and $\nu_{\mu}$ stand for the fields whose $P_{L}$ projections couple with $e$ and $\mu$ to the W-boson, we express $\nu_{e}$ and $\nu_{\mu}$ in terms of the "mass eigenfields" $\nu_{1}$ and $\nu_{2}$, and write $\nu_{e}=\cos \theta \nu_{1}+\sin \theta \nu_{2}$ and $\nu_{\mu}=-\sin \theta \nu_{1}+\cos \theta \nu_{2}$. Several examples of the coefficients $K$ and $F$ are tabulated for the $S M$ and two familiar extensions in Table (11). In the ultrarelativistic limit, an approximate Fock space of flavor states can be constructed 12, 13], while the $\left|\nu_{1}\right\rangle$ and $\left|\nu_{2}\right\rangle$ states propagate with the simple plane wave factors $\exp i\left(\mathbf{x} \cdot \mathbf{p}-E_{1,2} t\right)$, leading at order $\left(\Delta m^{2}\right) / E$ to the usual mass, energy and distance dependent argument of the oscillation probability. The task at hand is to fold this oscillation effect with the source and detector lepton-flavor violating effects to produce an "appearance probability" in terms of a convenient, uniform parameterization.

\section{Analysis of an Accelerator "Appearance" Pro- cess}

Consider $\pi^{+} \rightarrow \nu+\mu^{+}$decay to be the source of neutrinos, where the transition matrix element to a mass eigenstate $\mid \nu_{b}>$ reads $M_{b}^{S}=\left\langle\nu_{b}, \mu^{+}\left|L^{S}\right| \pi^{+}\right\rangle$, with $L^{S}$ given in Eq. (11). Consider the reaction $\nu+N_{i}(A, Z-1) \rightarrow N_{f}(A, Z)+e^{-}$to be the detection mechanism, with matrix element $M_{b}^{D}=<e^{-}, N_{f}\left|L^{S}\right| N_{i}, \nu_{b}>$. We may now write the $\mu \rightarrow e$ transition probability in the ultrarelativistic neutrino limit as 12, 13] $P_{\mu \rightarrow e} \sim\left|\Sigma_{b} M_{b}^{D} e^{-i E_{b} t} M_{b}^{S}\right|^{2}$, where the sum is over mass eigenstates and the equality holds to leading order in $m_{\nu} / E$. Spelling out this probability in the notation of Eq. (ID), we have the expression

$$
\begin{aligned}
P_{\mu \rightarrow e} \sim & \left(2^{3 / 2} G_{F}\right)^{4} \mid \Sigma_{b}\left(K_{A 2 j}^{h}\right)^{*} K_{B 1 k}^{h}\left(\bar{\mu} \Gamma_{A} P_{h} U_{j b} \nu_{b}\right)^{*} \times \\
& \bar{e} \Gamma_{B} P_{h} U_{k b} \nu_{b} e^{-i E_{b} t}<0\left|O_{A}\right| \pi^{+}><N_{f}\left|O_{B}\right| N i>\left.\right|^{2} .
\end{aligned}
$$

The sums over repeated indices $A, B, h, j, k$ and a sum over spins are implicit. In the same fashion as above, the $\mu^{+} \rightarrow e^{+}+\nu+\bar{\nu}$ process can be considered to 
be the source of neutrinos, with the effective Lagrangian $L^{L}$ replacing $L^{S}$ in the source matrix element. The "appearance" sought is then $\bar{\nu}+N_{i}(A, Z+1) \rightarrow$ $N_{f}(A, Z)+e^{+}$. The counterpart to Eq. (3) reads:

$$
\begin{aligned}
P_{\mu \rightarrow e} \sim & \left(2^{3 / 2} G_{F}\right)^{4} \Sigma_{c} \mid \Sigma_{d}\left(K_{B 1 k}^{h}\right)^{*} F_{A 2 j 1 m}^{h}\left(\bar{\mu} \Gamma_{A} P_{h} U_{j d} \nu_{d}\right) \times \\
& \left(\bar{e} \Gamma_{A} P_{h} U_{m c} \nu_{c}\right)^{*} e^{-i E_{d} t}\left(\bar{e} \Gamma_{B} P_{h} U_{k d} \nu_{d}\right)^{*}<N_{f}\left|O_{B}\right| N i>\left.\right|^{2} .
\end{aligned}
$$

The expression in Eq. (3) is appropriate to the LSND decay-in-flight (DIF) study and Eq. (位) to their decay-at-rest (DAR) study with $N_{i}=p$ and $N_{f}=n$. To make the notation more concrete, let us illustrate it with several examples.

\section{Purely Leptonic New Physics}

Our first example is applicable to the DAR situation, where $\mu^{+}$decay provides the $\bar{\nu}$ 's at the detector. We assume only the lepton source contains new, leptonnumber violating interactions, and that they are represented in the effective Lagrangian, Eq. (2), by $(V-A) \times(V-A)$ terms. The lepton-flavor violating case of interest to us has $i=2, j=1, k=1$ and $m=1,2$ or 3. Applying Eq. (4) to this situation, one reads off, for $\mu^{+} \rightarrow e^{+} \nu \bar{\nu}$ and $\bar{\nu}_{e}+N(Z) \rightarrow$ $e^{+}+N(Z-1)$,

$$
\begin{aligned}
P_{\mu \rightarrow e} \sim & \left\{\left(2^{3 / 2} G_{F}\right)^{4}\left|\bar{e} \gamma_{\lambda} P_{L} \nu<N_{f}\right| O_{V}^{\lambda}\left|N_{i}>\right|^{2}\left|K_{V 11}^{L}\right|^{2}\left|\bar{\mu} \gamma_{\sigma} P_{L} \nu \bar{\nu} \gamma_{\sigma} P_{L} e\right|^{2}\right\} \\
& \times \sum_{c}\left|\sum_{d} F_{V 2211}^{L} U_{2 d}^{*} e^{-i E_{d} t} U_{1 d} U_{1 c}^{*}+F_{V 211 m}^{L} U_{1 d}^{*} e^{-i E_{d} t} U_{1 d} U_{m c}^{*}\right|^{2} .
\end{aligned}
$$

With the new physics confined to the lepton-number violation in the $\mu^{+}$decay as described, we have $K_{V 11}^{L}=1=F_{V 2211}^{L}$ and $F_{V 211 m}^{L} \neq 0$. The factor in braces is exactly the one appropriate to the unsupressed sequence $\mu^{+} \rightarrow e^{+} \bar{\nu}_{\mu} \nu_{e}$ and $\bar{\nu}_{e}+N(Z) \rightarrow N(Z-1)+e^{+}$. The $P_{\mu \rightarrow e}$ probability can now be read off from Eq. (5), and for two flavor mixing we obtain

$$
\begin{aligned}
P_{\mu \rightarrow e} & =\sum_{m=e, \mu, \tau}\left[\tan ^{2} \psi_{m}+\left(1-\tan ^{2} \psi_{m}\right) \sin ^{2} 2 \theta \sin ^{2} x\right] \\
& +\left[\tan \psi_{e} \sin 4 \theta \sin ^{2} x\right. \\
& \left.+4 \tan \psi_{e} \sin 2 \theta \sin \varphi_{e} \sin x\left(\cos \varphi_{e} \cos x-\cos 2 \theta \sin \varphi_{e} \sin x\right)\right]
\end{aligned}
$$

where $2 x=\left(E_{2}-E_{1}\right) t$ and where $F_{V 211 m}^{L} \equiv \tan \psi_{m} e^{2 i \varphi_{m}}$ has been defined so that a uniform "all angles" representation of the appearance probability could be achieved. The limit $\varphi_{m} \rightarrow 0$, keeping only an $m=\mu$ value and keeping leading powers of $\psi_{m}<<1$, leads to the formula of Ref. [14] for the case that the direct lepton-flavor violation is only in the $\mu$-decay source interaction.

In Fig. (11) we show the $P_{\mu \rightarrow e}=0.003 \pm 0.001$ [3] contours in the $\sin ^{2} 2 \theta$, $\Delta M_{\mu e}^{2}$ parameter plane for $\psi_{e} \equiv F_{V 2111}^{L}$ values of 0 and 0.04 , where $\psi_{\mu}=\psi_{\tau}=$ 
Figure 1: The LSND $1 \sigma$ allowed region $(P=0.002-0.004$ for DAR) for no direct interaction new physics (solid curves) and purely leptonic new physics strength $\psi=0.04$ (dashed curves). Nonzero new physics phase makes the curves slightly lower for $\Delta M^{2}<1.8 \mathrm{eV}^{2}$. Also included are limits from Bugey (dash-dotted curve) and E776 (dotted curve).

Table 2: Relevant new physics couplings and upper bounds. Sources of the bounds are other than neutrino oscillation experiments.

\begin{tabular}{|l|c|c|}
\hline Coupling & Bound & Source \\
\hline$\left|\bar{h}_{11} \bar{h}_{21}^{*}\right| /\left(M_{\Delta_{L}^{2}} G_{F} 2 \sqrt{2}\right)$ & $7.6 \times 10^{-2}$ & $\nu e, \nu_{\mu} e, \bar{\nu} e$ \\
\hline$\left|\bar{h}_{11}\right|||_{22}^{*} \mid /\left(M_{\Delta_{L}^{2}} G_{F} 2 \sqrt{2}\right)$ & $1.1 \times 10^{-1}$ & $\mu \rightarrow e \nu \bar{\nu}$ \\
\hline$\left|\bar{h}_{11}\right|||_{23}^{*} \mid /\left(M_{\Delta_{L}^{2}} G_{F} 2 \sqrt{2}\right)$ & $9.1 \times 10^{-1}$ & $(g-2)_{\mu}$ \\
\hline$\left|\sum_{k} \lambda_{21 k}^{*} \lambda_{11 k} /\left(M_{\tilde{d}_{R}^{k}}^{2} G_{F} 2 \sqrt{2}\right)\right|$ & $2.5 \times 10^{-7}$ & $\mu T i \rightarrow e T i$ \\
\hline$\left|\lambda_{211}^{\prime *}\right|\left|\lambda_{231}\right| /\left(M_{\tilde{e}_{R}^{k}}^{2} G_{F} 2 \sqrt{2}\right)$ & $3.3 \times 10^{-2}$ & $\tau \rightarrow \ell \nu \bar{\nu}$ and $\pi \nu$ \\
\hline
\end{tabular}

$\varphi_{m}=0$ is assumed.5 The minimal $L-R$ model[15] is relevant to the case under consideration, since it generates lepton-flavor violating, purely leptonic, four-Fermi, $(V-A) \times(V-A)$ interactions by exchange of an iso-triplet Higgs particle. There is no $(V-A) \times(V-A) \mu^{+} \rightarrow e^{+}+\bar{\nu}_{e}+\nu_{x}$ effect in the $M S S M+\not R$ model, contrary to the assertion in Ref. [14]. In the notation of Eq. (11) we have $2^{2 / 3} G_{F} F_{V i j k m}^{L}=\frac{\bar{h}_{j k} \bar{h}_{i m}^{*}}{M_{\Delta_{L}}^{2}}$ as the identification of the relevant coefficients in the effective Lagrangian, Eq. (21). Yukawa coupling matrices are designated by $\bar{h}_{i j}=\frac{1}{2}\left(h_{i j}+h_{j i}\right)$ and $M_{\Delta_{L}}$ is the mass of the singly-charged member of the Higgs triplet, following the notation of Ref. [16]. The ranges of allowed values of $\frac{\bar{h}_{11} \bar{h}_{2 m}}{M_{\Delta}^{2}}$ for the $L-R$ model are shown in Table (2). It is surprising that a considerable flexibility in choosing relevant $L-R$ model parameters is still allowed by all experimental constraints.

\section{The Same New Physics at Source and Detec- tor}

A case that injects the same new semileptonic physics at source and detector in the DIF situation is exemplified by the pure $(V-A) \times(V-A)$ choices $K_{V 22}^{L}=K_{V 11}^{L}=1$, which follow from the standard model, and $K_{V 21}^{* L}=K_{V 12}^{L} \equiv$

\footnotetext{
${ }^{5}$ Since we are showing only trends and not fits, we adopt the Gaussian "toy model" used for illustrating oscillations in Ref. 20] with $b_{0}$ and $\sigma_{b}$ appropriate to LSND.
} 
Figure 2: The LSND $1 \sigma$ allowed region $(P=0.0015-0.0037$ for DIF) for no new direct interaction physics (solid curves) and for $P=0.0026$ with $\psi=0.01$ (dotted curve), $\psi=0.02$ (dot-dashed curve) and $\psi=0.03$ (dashed curve) when there is the same new physics at the source and detector.

$\tan \psi e^{2 i \varphi}$ which defines the new physics. 5 The $P_{e \rightarrow \mu}$ expression that results when two-flavor mixing is assumed reads

$$
P_{\mu \rightarrow e}(D I F)=4 \tan ^{2} \psi \cos ^{2} x+\sin ^{2} 2 \theta \sin ^{2} x-2 \tan \psi \sin 2 \theta \sin 2 \varphi \sin 2 x
$$

for DIF.7 On the other hand,

$$
\begin{aligned}
P_{\mu \rightarrow e}(D A R) & =\tan ^{2} \psi+\sin ^{2} 2 \theta \sin ^{2} x\left(1-\tan ^{2} \psi\right) \\
& +\tan \psi \sin 2 \theta\left[-\sin 2 \varphi \sin 2 x+2 \cos 2 \varphi \cos 2 \theta \sin ^{2} x\right]
\end{aligned}
$$

for DAR, where only the detection is affected by the new interaction. In writing Eq. (8), we have assumed that there is no lepton-flavor violating new physics interaction in $\mu$-decay. If $\sin ^{2} x<<\sin ^{2} \psi$, then $P_{\mu \rightarrow e}(D I F) \simeq 4 P_{\mu \rightarrow e}(D A R)$, so a combination of $L^{S}$ and $L^{L}$ effects would have to be invoked to produce the $P_{\mu \rightarrow e}(D I F) \simeq P_{\mu \rightarrow e}(D A R) \simeq 0.003$ result reported by the two LSND experiments. Fig. (2) shows plots of the $P_{\mu \rightarrow e}(D I F)=0.0026 \pm 0.0011$ boundaries in the $\Delta M_{\mu e}^{2} \leftrightarrow \sin ^{2} 2 \theta$ parameter plane for several choices of $\psi$ with $\varphi=0$. For smaller $\psi$ values, $\psi \lesssim 0.02$, the modifications to the $\psi=0$ curve have the same general character as those of Fig. (11), moving the curves leftward and downward. A dramatic change occurs when $\psi \gtrsim 0.03$, however. In Eq. (7) the large term $4 \tan ^{2} \psi \cos ^{2} x$ tends to "overshoot" the input value of $P_{\mu \rightarrow e}$ as $\tan ^{2} \psi$ grows, so $x$ needs to increase to compensate this growth. But the second term grows as $x$ grows, so $\sin ^{2} 2 \theta$ must control this growth and therefore a maximum $\sin ^{2} 2 \theta$ value is established for larger $\tan ^{2} \psi$ values. It is the factor four in the first term of Eq. (7), reflecting the compound effects of source and detector, that produces the new effect at rather modest $\tan ^{2} \psi$ values.

To make the connection to the minimal $\not R$ SUSY model, 18 we read off $K_{V 21}^{* L}=K_{V 12}=\sum_{k} \frac{\lambda_{21 k}^{\prime *} \lambda_{11 k}^{\prime}}{M_{\tilde{d}_{R}^{k}}^{2}} \cdot \frac{1}{2^{3 / 2} G_{F}}$ from the semileptonic effective Lagrangian that follows from exchange of the $\tilde{d}_{R}^{k}$ squark, with $k$ the family index, in the notation of Ref. [19]. In this model, the neutral current $e \leftrightarrow \mu$ transition operator has the same coefficient, and the bound from $\mu T i \rightarrow e T i[21$, 22] is severe, as listed in Table (2), making this case academic in the minimal $\not R$ SUSY model. At the moment we cannot offer a well-motivated model with the

\footnotetext{
${ }^{6}$ We use the same symbols $\psi$ and $\varphi$ to parameterize the different cases for economy of notation. The context makes clear that these are independent parameters for the different cases.

${ }^{7}$ On this point, we completely disagree with Ref. [14], which asserts that there is no effect when the physics at source and detector are the same.
} 
structure $K_{V 21}^{* L}=K_{V 12}^{L}$, but we present the above to illustrate the structure of the case where the source and detector new physics is the same.

\section{New Physics Only at the Detector}

Because the DAR and DIF experiments of the LSND collaboration have different backgrounds and different systematic errors, the reported agreement between the results of the two experiments suggests a common origin[ [ 4 . We point out here that a lepton-number violating effective interaction that is only operative in the detection processes tends to produce this effect.

Consider the effective Lagrangian case with $K_{V 22}^{L}=K_{L 11}^{L}=1$ for the standard model, $K_{V 12}^{L} \neq 0=\tan \psi e^{2 i \varphi}$ and all other coefficients negligible for the new physics. We find that, in the two-neutrino mixing case, the same parameterization, Eq. (8), applies to both the DAR and DIF cases even with the direct, lepton-number violating interactions effects combined with those of the oscillations. In principle the agreement between the DAR and DIF measured $\nu_{\mu} \leftrightarrow \nu_{e}$ oscillation probabilities could be represented by $\tan ^{2} \psi$, a purely direct interaction effect with $\tan ^{2} \psi \simeq 0.003$

In surveying our example models, the minimal $S U_{L}(2) \times S U_{R}(2) \times U(1)_{B-L}$ weak interaction model and the MSSM without $R$-parity, one finds that the above parameter choice $K_{V 12 \neq 0}^{L}$ and all other new physics $K_{A i j}^{L}=0$, does not occur. The instructive case $K_{S 12}^{L} \neq 0, K_{S 21}^{L}=0$ and with $K_{V i j}^{L}=0$ by choice, does arise from the $R$-parity violating interactions, however, and we summarize this case next.

\section{Different New Physics Lorentz Structures}

Up to this point, we have considered only cases where the new interaction, charged current effective four-Fermi Lagrangian has the same $(V-A) \times(V-A)$ structure as the standard model. This ensured all matrix elements of interest had the same structure and the dynamics of the source and detector physics could be lumped into an overall factor that is normalized away in the definition of the lepton "flavor change", or "oscillation" probability. If the Lorentz structure of the charged current $\times$ charged current interaction is not the same as that of the standard model, then the analysis of $P_{\mu \rightarrow e}$ necessarily brings in details of the matrix elements at the source and/or detector.

In the situation mentioned above where $K_{S 12}^{L} \neq 0$ is the only relevant new physics, and the scalar interaction is of the form $(S-P)_{\text {lepton }} \times(S+P)_{\text {quark }}$, the matrix elements of the new physics and those of the standard model operators are distinctly different. Dividing out a common factor from the source, the DAR 
case looks schematically like, in the two-flavor mixing case,

$$
\begin{aligned}
P_{\mu \rightarrow e} & \sim\left|\bar{e} \gamma_{\lambda} P_{L} \nu<N_{f}\right| O^{\lambda} \mid N_{i}>\left(-\sin 2 \theta \sin x e^{i x}\right) \\
& +K_{S 12}^{L} \bar{e} P_{L} \nu<N_{f}\left|O_{S}\right| N_{i}>\left.\left(1-2 \cos ^{2} \theta \sin x e^{i x}\right)\right|^{2}
\end{aligned}
$$

The new physics, lepton-number violating interaction effects depend both on the parameters $\left(K_{S 12}^{L}, \theta\right.$ and $\left.x\right)$ and on the comparative role of the matrix elements: $\bar{e} \gamma_{\lambda} P_{L} \nu<N_{f}\left|O^{\lambda}\right| N_{i}>$ in the standard model term and $\bar{e} P_{L} \nu<N_{f}\left|O_{S}\right| N_{i}>$ in the new interaction. With $O_{S}=\bar{u} P_{R} d$, as in the $R$-parity violating model where $K_{S 12}^{L}=\lambda_{311}^{\prime} \lambda_{231} /\left(M_{\tilde{e}_{3}}^{2} G_{F} 2 \sqrt{2}\right), K_{S 21}^{L}=0$ and $K_{V 12}^{L}=K_{V 21}^{* L}<<K_{S 12}^{L}$, we can evaluate the spin-averaged transition probability for a $\nu+n \rightarrow e+p$ detector transition to illustrate the DAR application. Simplifying the $\beta$-decay matrix element to pure $V-A$ for illustration purposes, we find

$$
P_{\mu \rightarrow e} \sim \sin ^{2} 2 \theta \sin ^{2} x+\left|K_{S 12}^{L}\right|^{2} \frac{t\left(t-2 M_{N}^{2}\right)}{4\left(s-M_{N}^{2}\right)^{2}}\left(1-\sin ^{2} 2 \theta \sin ^{2} x\right),
$$

where $M_{N}$ is the mass of the nucleon, $s=\left(P_{\nu}+P_{n}\right)^{2}$ and $t=\left(P_{\nu}-P_{e}\right)^{2}$. In writing Eq. (10) we have dropped the cross term, which is proportional to $m_{e}$, and we have not displayed a final state phase space integration. The matrix element effects are clearly crucial, as is shown by the suppression of the cross term and the nontrivial nature of the relative kinematical factor between the $(V-A) \times(V-A)$ standard model structure and the $(S-P) \times(S+P)$ new physics structure. For energies appropriate to LSND, the kinematical factor ratio that multiplies the new physics term is small (of order $\left|t / m_{N}^{2}\right| \sim$ $\left.(30 \mathrm{MeV} / 1 \mathrm{GeV})^{2}\right)$, so it is unlikely that such terms can contribute significantly to a $P_{\mu \rightarrow e}$ signal. For completeness, we mention that the MSSM with $\not R$, where $\left|\lambda_{311}^{\prime *} \lambda_{231}\right| / M_{\tilde{e}_{3}}^{2} G_{F} 2 \sqrt{2} \leq 2 \times 10^{-4} 22$, requires a kinematical enhancement to bring it up to the order of $10^{-3}$ relevant to the LSND reported result. The important lesson to stress, however, is that one must look at the detailed matrix element evaluations to draw conclusions in these cases where the Lorentz structure of the effective Lagrangian's new physics terms is different from that of the standard model.

\section{Three-Family Mixing Application}

Our illustrations so far have relied on two-family mixing parameterizations, which are appropriate to the $M_{3}>>M_{2}>>M_{1}$ type of hierarchy of neutrino masses. The framework we present applies to any number of families, so we discuss a simple, "almost viable" version of three-family mixing as our last illustration [6]. The starting, simplifying assumption is that $M_{1} \sim M_{2}<<$ $M_{3}$, which reduces the three-family mixing to a "one-scale" problem; namely $\Delta M_{13}^{2} \simeq \Delta M_{23}^{2} \equiv \Delta M^{2}$ with $\Delta M_{12}^{2} \simeq 0$. Returning to the DAR case with new 
Figure 3: For three-flavor, one-scale mixing, the LSND $1 \sigma$ allowed region (DAR) for no new direct interaction physics (solid curves) and purely leptonic new physics strength $\psi=0.02$ (dotted curves) and $\psi=0.04$ (dashed curves).

physics at the source and a restriction to $m=1$ in the flavor sum to reduce clutter in the notation, we have

$$
P_{e_{\mu}}=\left|-2 i U_{23}^{*} U_{13} \sin x e^{-i x}+\tan \psi e^{2 i \varphi}\left(1-2 i\left|U_{13}\right|^{2} \sin x e^{-i x}\right)\right|^{2},
$$

where $F_{V 2111} \equiv \tan \psi e^{2 i \varphi}$. With the parameterization $U_{23}=\sin \theta_{23} \cos \theta_{13}$ and $U_{13}=\sin \theta_{13} e^{-i \delta_{13}}$ [20], one can write out an expression similar to, but even more obscure than Eq. (8). The form shown in Eq. (11) makes the point that the first term alone gives the result of Ref. [6], and the new interactions produce a leading $\tan ^{2} \psi$ term plus other terms similar to those in Eq. (8). The flexibility afforded by the inclusion of the direct interaction is shown in Fig. (3), where the effect of increasing $\psi$ on the $\tan ^{2} \theta_{13}$ vs. $\tan ^{2} \theta_{23}$ plot is shown to be significant for $\psi \simeq 0.04$.

\section{Discussion}

We have developed and applied a compact analysis that treats neutrino flavorchanging phenomena - oscillations plus direct interactions - in a unified manner. We showed standard oscillation parameter plots can be shifted significantly for rather modest values of $\psi$, an angle parameterizing direct interactions. We also showed the positive DAR result reported by LSND[3] can be accounted for by direct interaction effects in the $L-R$ model.

Open possibilities for further applications include the unified treatment of the neutrino masses and direct interactions within a model like the $M S S M+\not R$ model and the applications to general three-family and three-family plus sterile neutrino pictures.

Whether direct lepton-flavor changing effects turn out to be important or not, the framework offered here should provide an efficient means to survey the possibilities.

\section{Acknowledgements}

We thank Kerry Whisnant and Herman Munczek for discussions and Jack Gunion and Tao Han for a timely $\not R$ suggestion. L.M.J. acknowledges support from the University of Kansas Dissertation Year program. This work was supported in part by U.S. DOE Grant No. DE-FG02-85ER40214. 


\section{References}

[1] Homestake Collaboration, B.T. Cleveland et al., Nucl. Phys. B. (Proc. Suppl.) 38, (1995) 47; Kamiokande II Collaboration, K.S. Hirata, et al., Phys. Rev. D 44, (1991) 2241; Kamiokande III Collaboration, Y. Fukuda et al. Phys. Rev. Lett. 77 (1996) 1683; SAGE Collaboration, W. Hampel et al., Phys. Lett. B 388 (1996) 384; Y. Tatsuka in Proceedings of the XVIII International Symposium on Lepton Photon Interactions, (July 28-Aug. 1, 1997 Hamburg). Unpublished.

[2] Kamiokande Collaboration, H.S. Hirata et al., Phys. Lett. B280 (1992) 146; Y. Fukuda et al., Phys. Lett. B335 (1994) 237; R. Becker-Szendy et al., Phys. Rev. D46 (1992) 3720; Frejus Collaboration, Ch. Berger, et al., Phys. Lett. B227 (1984), 489; NUSEX Collaboration, M. Aglietta et al., Europhys. Lett. 8, 611 (1989); Y. Tatsuka in Proceedings of the XVIII International Symposium on Lepton Photon Interactions, (July 28-Aug. 1, 1997, Hamburg) Unpublished; Soudan Collaboration, W.W.M. Allison et al., Phys. Lett. B391 (1997) 491.

[3] LSND Collaboration, C. Athanassopoulos et al., Phys. Rev. Lett. 77 (1996) 3082; C. Athanassopoulos et al., Phys. Rev. C 54 (1996) 2685.

[4] LSND Collaboration, C. Athanassopoulos et al., nucl-ex/9706006.

[5] KARMEN Collaboration, G. Drexlin Nucl. Phys (Proc. Suppl.) B 38 (1995) 235; E776 Collaboration, L. Borodovsky et al., Phys. Rev. Lett. 68 (1992) 274 .

[6] C. Cardall and G. Fuller, Phys. Rev. D 53 (1996) 4421.

[7] G. Fogli, E. Lisi and G. Scioscia, Phys. Rev. D. 56 (1997) 3081.

[8] V. Barger, T. Weiler and K. Whisnant, hep-ph/9712495 and references therein (for papers that add a fourth "sterile" neutrino).

[9] N. Hata and P. Langacker, Phys. Rev. D 56 (1997) 6107; Y. Tatsuka, Ref. 1; C. Yanagisawa, talk given at PHENO 98, Madison, March 1998.

[10] L. Wolfenstein, Phys. Rev. D 17 (1978) 2369; ibid 20 (1979) 2634; S. Mikheyev and A. Smirnov, Yad. Fiz. 42 (1985) 1441 [Sov.J.Nucl.Phys. 42 (1985) 913].

[11] Y. Tatsuka, Ref. [2]; M. Gonzalez-Garcia, H. Nunokawa, O. Peres, T. Stanev and J. Valle, hep-ph/9801368.

[12] C. Giunti, C. Kim and U. Lee, Phys. Rev. D 45 (1992) 2414, give a simple and clear discussion strictly within the neutrino oscillation possibility. 
[13] C. Giunti, C. Kim, J. Lee and U. Lee, Phys. Rev. D 48 (1993) 4310; K. Kiers and N. Weiss, Phys. Rev. D 57 (1998) 3091; C. Giunti and C. Kim, hep-ph/9711363.

[14] Y. Grossman, Phys. Lett. B 359 (1995) 141.

[15] R. Mohapatra and G. Senjanovic, Phys. Rev. D 23 (1981) 165.

[16] N. Deshpande, J. Gunion, B. Kayser and F. Olness, Phys. Rev. D 44, (1991) 837.

[17] S. Weinberg, Phys. Rev. D 26 (1982) 287; N. Sakai and T. Yanagida, Nucl. Phys. B 197 (1982) 133.

[18] For a recent review, see H. Dreiner, hep-ph/9707435.

[19] V. Barger, G. Giudice and T. Han, Phys. Rev. D 40 (1989) 2984.

[20] Particle Data Group, R.M. Barnett et al., Phys. Rev. D 54 (1996) 1.

[21] J. Kim, P. Ko and D.-G. Lee, Phys. Rev. D 56 (1997) 100.

[22] P. Roy, hep-ph/9712520 and references therein. 


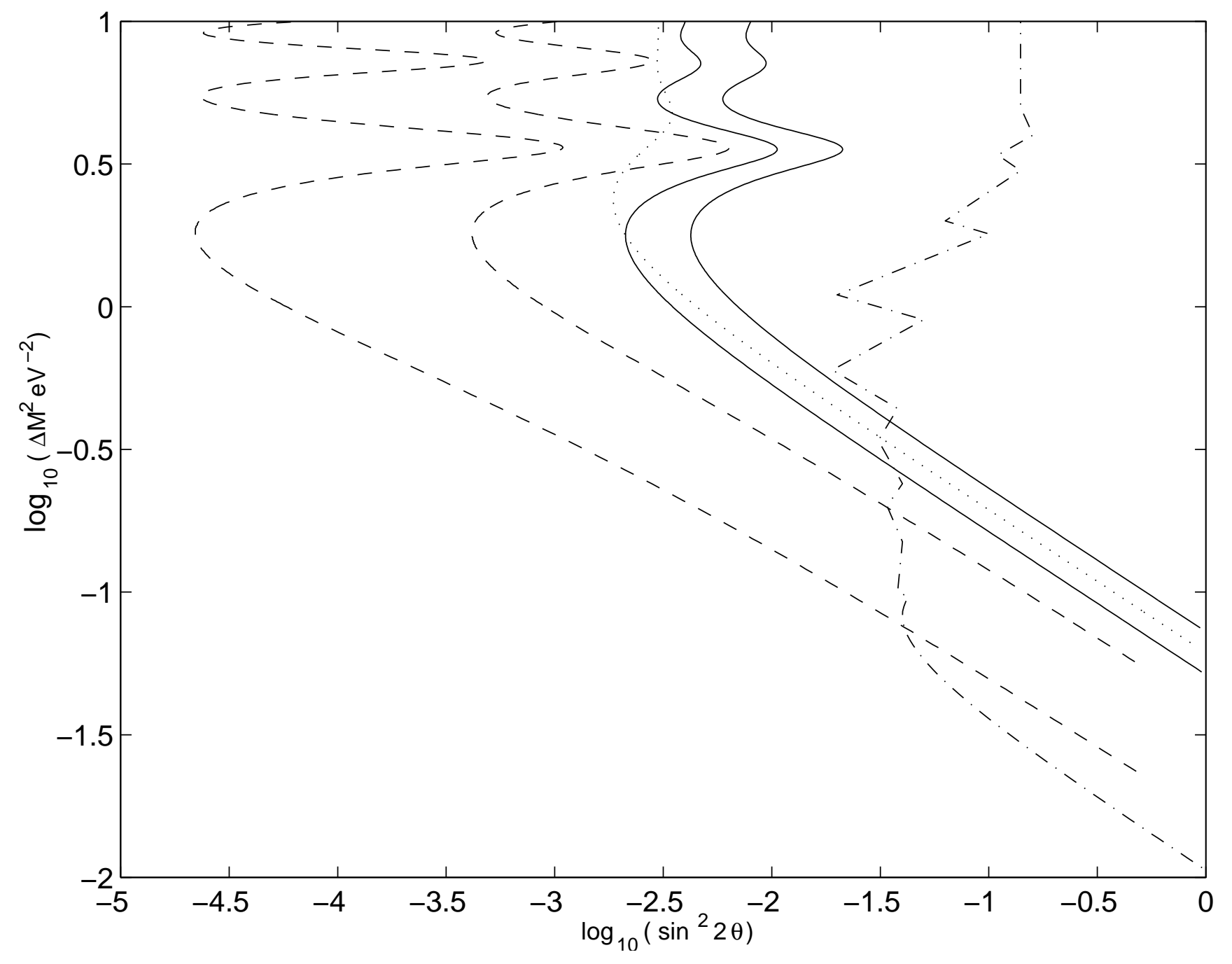




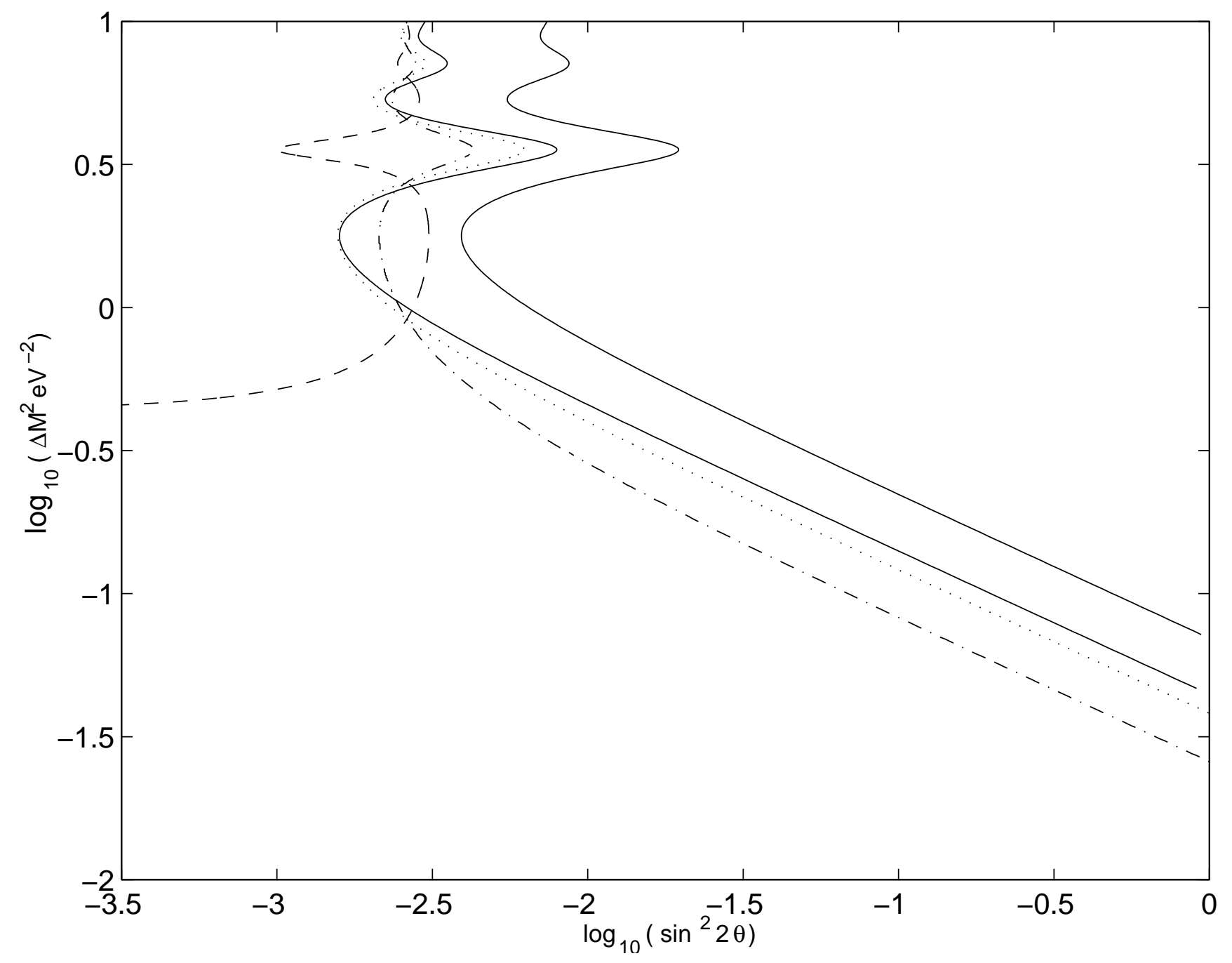




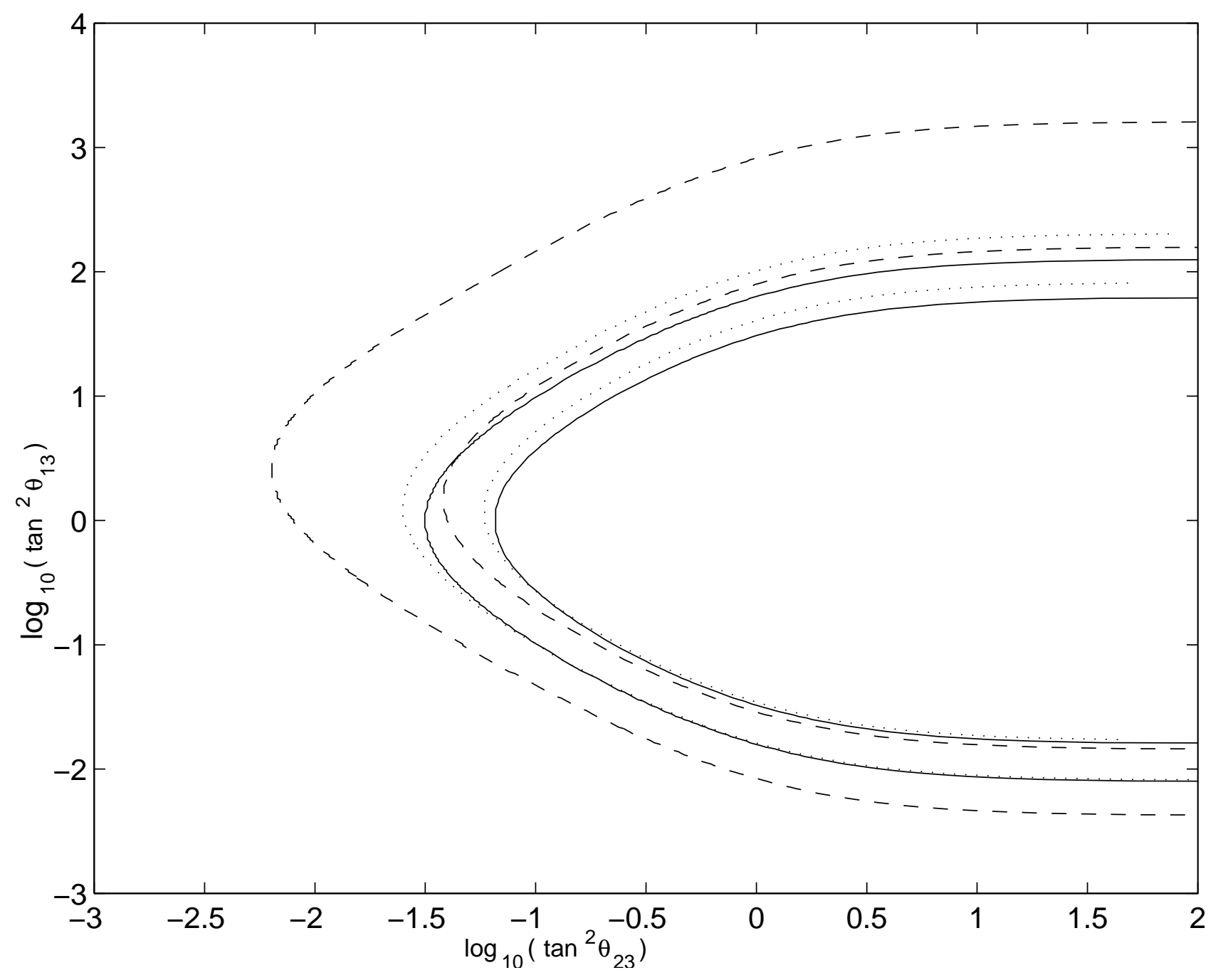

